Rapid Reviews COVID-19

\title{
Review 1: "Disparities in \\ COVID-19 Fatalities among \\ Working Californians"
}

\section{Isobel Routledge ${ }^{1}$}

${ }^{1}$ UCSF

Published on: Dec 28, 2021

License: Creative Commons Attribution 4.0 International License (CC-BY 4.0). 


\section{$\underline{\text { RR:C19 Evidence Scale rating by reviewer: }}$}

- Reliable. The main study claims are generally justified by its methods and data. The results and conclusions are likely to be similar to the hypothetical ideal study. There are some minor caveats or limitations, but they would/do not change the major claims of the study. The study provides sufficient strength of evidence on its own that its main claims should be considered actionable, with some room for future revision.

$* * * * * * * * * * * * * * * * * * * * * * * * * * * * * * * * * * * * * * *$

\section{Review:}

This work estimates COVID-19 mortality rates among working or likely working Californians, and describes the demographic characteristics of these individuals, as well as calculating mortality rates amongst different employment sectors and demographic groups. The study reports an overall age-adjusted COVID-19 mortality rate of 30.0 per 100,000 workers (95\% confidence interval, 29.3-30.8), and estimated higher mortality rates in particular occupations such as farming, material moving and construction. The study also reported mortality rates which were three-fold higher for male compared with female workers and three- to seven-fold higher for Latino and Black workers compared with Asian and White workers.

To reach these findings, the authors linked three statewide data sources: the Electronic Death Registration System (EDRS); the COVID-19 Case Registry; and the Employment Development Department (EDD) records as well as using 2019 data from the American Community Survey to calculate denominator populations. Although there are caveats to generating mortality rates from these types of record data, the authors have been very clear about these limitations and they would not affect the main conclusions of the study. Nonetheless, these limitations should be taken into consideration when interpreting the results - for example mortality rates rely on 2019 American Community Survey (ACS) microdata to estimate the denominators, and these data are both slightly outdated and affected by non-response biases. In addition, as pointed out by the study authors, COVID-19 deaths where the patient did not undergo PCR testing were excluded, meaning that mortality rates may be underestimated, particularly for medically underserved communities.

The use of a 'likely working' category (where there is no confirmed occupation within the EDD records but no clear indication of not working in other records) captures 
those who may be undocumented, working in the informal economy, or otherwise not captured by employment records. The authors note that "over $60 \%$ of the 'likely working' decedents were foreign-born and nearly a third lacked a valid social security number, suggesting that this group included undocumented immigrants." It is important to capture this group of workers, who may be more vulnerable from both an employment and healthcare access perspective. I agree that it is likely that undocumented workers were captured in this population, however it is speculative and potentially misleading to focus on this, as social security numbers may be missing through error or other reasons. This group does mainly contain individuals who do have social security numbers and may be missing from the EDD database for other reasons.

As a result of the limitations inherent in the data available I have lower confidence in the specific mortality rates reported, but the broad findings and conclusions appear robust and supported by the data and approach. This manuscript generally confirms previous findings related to COVID-19 health disparities, although the study did not find above-average COVID-19 mortality in healthcare occupations overall, which is in contrast to findings from studies carried out in other geographic locations including the United Kingdom and Massachusetts, which the authors hypothesise may be explained by the timing of outbreak onset in the other settings, which were badly affected very early in the pandemic when less personal protective equipment was available and the mode of transmission of SARS-CoV-2 was more poorly understood.

This work is clearly written, with clear recommendations for prioritisation of interventions to the highest mortality occupation groups. The authors cite relevant literature and do not overstate their conclusions. However, to improve transparency and clarity, although the authors describe much of the process in words, it would be helpful to have a diagram showing the pipeline for how data were included/excluded and linked, with sample sizes at each stage. The work has received ethical approval and addresses important health disparities; I do not have any concerns about the article from a DEI/ethical perspective. 